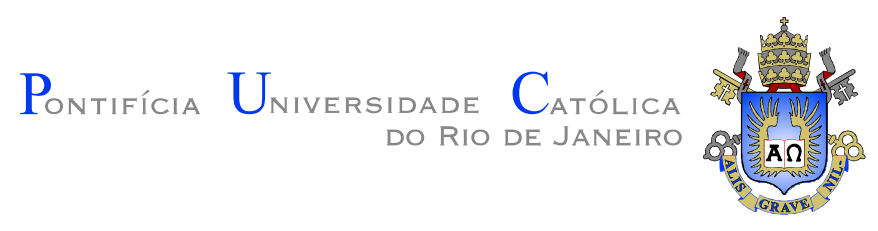

Julio César Álvarez Iglesias

\title{
Desenvolvimento de um sistema de microscopia digital para classificação automática de tipos de hematita em minério de ferro
}

Tese apresentada como requisito parcial para obtenção do título de Doutor pelo Programa de Pós-graduação em Engenharia de Materiais e de Processos Químicos e Metalúrgicos do Departamento de Engenharia de Materiais da PUC-Rio.

Orientador

Prof. Sidnei Paciornik

Co-Orientador: Dr. Otávio da Fonseca Martins Gomes

Rio de Janeiro

Agosto de 2012 

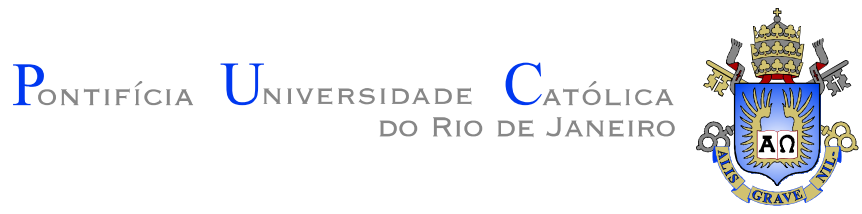

\section{Julio César Álvarez Iglesias}

\section{Desenvolvimento de um sistema de microscopia digital para classificação automática de tipos de hematita em minério de ferro}

Tese apresentada como requisito parcial para obtenção do título de Doutor pelo Programa de Pós-graduação em Engenharia de Materiais e de Processos Químicos e Metalúrgicos da PUC-Rio. Aprovada pela Comissão Examinadora abaixo assinada.

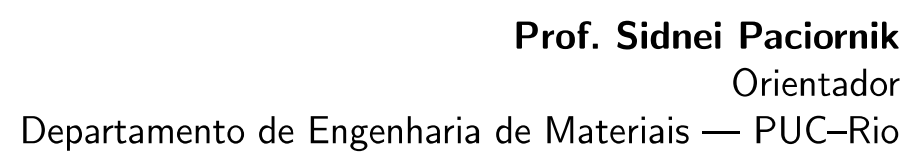

Dr. Otávio da Fonseca Martins Gomes Co-Orientador Centro de Tecnologia Mineral - CETEM/MCTI

Prof. Paulo Roberto Gomes Brandão Universidade Federal de Minas Gerais - UFMG

Prof. Leonardo Evangelista Lagoeiro Universidade Federal de Ouro Preto - UFOP

Dr. Reiner Neumann Centro de Tecnologia Mineral - CETEM/MCTI

Dr. Marcos Henrique de Pinho Maurício Departamento de Engenharia de Materiais - PUC-Rio

Prof. José Eugenio Leal Coordenador Setorial do Centro Técnico Científico - PUC-Rio 
Todos os direitos reservados. É proibida a reprodução total ou parcial do trabalho sem autorização da universidade, do autor e do orientador.

\section{Julio César Álvarez Iglesias}

Graduou-se em Física pela Universidade da Havana (Havana, Cuba). Fez mestrado no Departamento de Engenharia de Materiais da PUC-Rio, especializando-se na área de microscopia digital, processamento e análise digital de imagens. Desenvolveu um grande número de rotinas de processamento digital de imagens para a companhia Vale na identificação e caracterização de minério de ferro assim como seus aglomerados.

Ficha Catalográfica

Álvarez Iglesias, Julio César

Desenvolvimento de um sistema de microscopia digital para classificação automática de tipos de hematita em minério de ferro / Julio César Álvarez Iglesias; orientador: Sidnei Paciornik; co-orientador: Otávio da Fonseca Martins Gomes. - Rio de Janeiro : PUC-Rio, Departamento de Engenharia de Materiais, 2012.

136 f.: il.(color.) ; $30 \mathrm{~cm}$

1. Tese (doutorado) - Pontifícia Universidade Católica do Rio de Janeiro, Departamento de Engenharia de Materiais, 2012.

Inclui bibliografia.

1. Engenharia de Materiais - Tese. 2. Minério de Ferro; 3. Cristais de Hematita;. 4. Microscopia Digital;. 5. Análise de Imagens; . 6. Classificação; . 7. Microscopia de Luz Polarizada.. I. Paciornik, Sidnei. II. da Fonseca Martins Gomes, Otávio. III. Pontifícia Universidade Católica do Rio de Janeiro. Departamento de Engenharia de Materiais. IV. Título.

CDD: 620.11 


\section{Agradecimentos}

Em primeiro lugar, gostaria de agradecer especialmente a meu orientador Prof. Sidnei Paciornik e co-orientador Dr. Otávio Gomes. Em particular, quero manifestar o meu apreço ao Sidnei que, além de orientador, tem sido um amigo. Eu não tenho palavras para expressar minha sincera gratidão a ele, mas eu me considero afortunado por ter trabalhado com ele nos últimos seis anos; dois deles durante o meu mestrado. Agradecimentos especiais ao meu co-orientador Otávio Gomes por ter me guiado durante esta caminhada com seu extenso conhecimento e entusiasmo.

Eu tenho a sorte de ter a oportunidade de colaborar com colegas de laboratório muito competentes que sem dúvida têm contribuído significativamente para essa tesse. Eles também têm sido os responsáveis por criar um ambiente agradável e estimulante para trabalhar durante esses quatro anos de minha pesquisa. Individualmente, gostaria de agradecer a Karen Augusto por ter sacrificado longas horas na coleta de dados e análise de vários resultados importantes para a minha tese, a David Pirotte pelo suporte com as questões técnicas relacionadas ao uso do Lattex, a Debora Turon pelos momentos compartilhados e aprendizado dividido, assim como a Luciana Carneiro.

Eu também devo muito a Maria Beatriz Vieira, nossa colaborada da Vale, que gerenciou e nos permitiu o acesso a este desafiante problema. Certamente contar com a sua colaboração foi decisiva para o impacto e contribuição da minha tese no contexto da indústria brasileira.

Agradeço ao CETEM/MCTI pela parceria e pelo apoio na aquisição e processamento dos resultados usados nesta tese.

Também sou grato aos membros da minha banca examinadora, Prof. Paulo Roberto Gomes Brandão, Prof. Leonardo Evangelista Lagoeiro, Dr. Reiner Neumann e Dr. Marcos Henrique de Pinho Maurício, que comprometeram muito generosamente seu tempo e conhecimento para avaliar a minha tese.

Eu também gostaria de agradecer à minha esposa, por cada minuto de amor, estímulo, paciência, encorajamento e força. A meus pais pelo apoio, mesmo a muitos quilômetros de distância, que tem sido essencial para encontrar forças e continuar lutando pelos meus objetivos. Palavras não podem expressar a imensidão da gratidão que tenho por eles. À minha família em geral pela força e confiança.

Por último, mas não menos importante, gostaria de agradecer à CAPES pelo apoio financeiro e à PUC-Rio pela bolsa de isenção de mensalidades do doutorado. 


\section{Resumo}

Álvarez Iglesias, Julio César; Paciornik, Sidnei; da Fonseca Martins Gomes, Otávio. Desenvolvimento de um sistema de microscopia digital para classificação automática de tipos de hematita em minério de ferro. Rio de Janeiro, 2012. 136p. Tese de Doutorado - Departamento de Engenharia de Materiais, Pontifícia Universidade Católica do Rio de Janeiro.

O minério de ferro é um material policristalino oriundo de processos naturais complexos durante tempos geológicos, que dão origem a características intrínsecas e comportamento industrial variado. A grande maioria dos minérios de ferro brasileiros é essencialmente hematítico. A hematita pode ser classificada como lobular, lamelar, granular, microcristalina ou martita. $\mathrm{Na}$ indústria mineral, esta caracterização é tradicionalmente realizada por operadores humanos a partir da observação de amostras no microscópio ótico, sujeita a grandes variações. Assim, é relevante desenvolver um procedimento que permita a discriminação dos diferentes tipos de hematita e a medida de características tais como o tamanho de cristal. Esta tese propõe um sistema que mede e classifica automaticamente tipos texturais de hematita baseado no processamento e na análise de imagens de microscopia ótica, em campo claro, polarização linear e polarização circular. Foram desenvolvidas rotinas para aquisição, registro, segmentação, reconhecimento e análise morfológica de cristais de hematita. A segmentação automática de cristais de hematita foi baseada no cálculo da distância espectral, a fim de controlar o crescimento de regiões partindo das sementes. Os resultados da identificação dos cristais obtidos, tanto nas imagens obtidas com polarização linear quanto com polarização circular, foram muito promissores. Atributos de tamanho e forma dos cristais identificados foram obtidos. Estes dados foram usados como conjunto de treinamento para classificadores supervisionados, permitindo reconhecer as classes de hematita granular, lamelar e lobular. Taxas de acerto globais próximas a $98 \%$ foram obtidas, tanto para autovalidação, quanto para a validação cruzada.

\section{Palavras-chave}

Minério de Ferro; Cristais de Hematita; Microscopia Digital; Análise de Imagens; Classificação; Microscopia de Luz Polarizada. 


\section{Abstract}

Álvarez Iglesias, Julio César; Paciornik, Sidnei; da Fonseca Martins Gomes, Otávio. Development of a digital microscopy system for automatic classification of hematite types in iron ore.. Rio de Janeiro, 2012. 136p. PhD Thesis - Departamento de Engenharia de Materiais, Pontifícia Universidade Católica do Rio de Janeiro.

Iron ore is a polycrystalline material created by complex natural processes during geological periods, which give rise to intrinsic characteristics and varied industrial behavior. The vast majority of the Brazilian iron ores belong essentially to the hematitic type. Hematite can be classified as lobular, lamelar, granular, micro-crystalline or martite. In the mineral industry, the characterization of iron ore and its agglomerates is traditionally developed by human operators from the observation of samples under the optical microscope, which may suffer large variations. Thus, it is important to develop a procedure that allows the discrimination of the different hematite types and the measurement of characteristics such as crystal size. The present thesis proposes a system for the automatic classification of hematite textural types, based on digital processing and analysis of optical microscopy images, in bright field, linear and circular polarized light. Routines were developed for the acquisition, registration, recognition and morphological analysis of hematite crystals. The automatic segmentation of hematite crystals was based on calculating the spectral distance, in order to control the region expansion from the seeds. The results regarding the identification of the obtained crystals were very promising. Size and shape attributes were obtained and used as a training set for supervised classifiers, leading to the recognition of granular, lamelar and lobular hematite classes. Global success rates close to $98 \%$ were obtained concerning self-validation as well as crossed validation.

\section{Keywords}

Iron Ore; Hematite Crystals; Digital Microscopy; Image Analysis; Classification; Polarized Light Microscopy. 


\section{Sumário}

$\begin{array}{lr}\text { Lista de Abreviaturas } & 13\end{array}$

1 Introdução 14

2 Revisão Bibliográfica $r$

2.1 Minério de Ferro 18

2.1.1 Hematita 22

2.2 Microscopia Digital 25

2.2.1 Polarização da Luz 27

2.3 Processamento e Análise Digital de Imagens 33

2.3.1 Pré-processamento 34

2.3.1.1 Registro de imagem $\quad 34$

$\begin{array}{lll}\text { 2.3.1.2 Delineação } & 37\end{array}$

$\begin{array}{lll}2.3 .1 .3 & \text { Filtro Mediana } & 39\end{array}$

2.3.2 Técnicas de segmentação 41

2.3.2.1 Limiarização 42

2.3.2.2 Segmentação por Contornos 44

2.3.2.3 Crescimento de Regiões $\quad 45$

2.3.3 Pós-processamento 48

2.3.3.1 Operações Lógicas $\quad 48$

2.3.3.2 Operações Morfológicas $\quad 50$

2.3.4 Extração de Atributos $\quad 57$

2.3.5 Reconhecimento e Classificação 63

2.3.5.1 Conjunto de treinamento $\quad 65$

2.3.5.2 Conjunto de características $\quad 66$

$\begin{array}{lll}\text { 2.3.5.3 Classificadores } & 67\end{array}$

3 Materiais e Métodos $\quad 71$

3.1 Seleção e Preparação de Amostras $\quad 71$

3.2 Aquisição de Imagens ao Microscópio Ótico 72

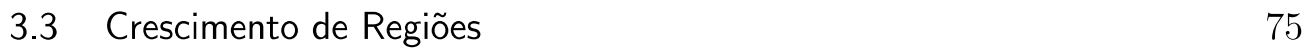

$\begin{array}{lll}\text { 3.3.1 Pré-processamento } & 75\end{array}$

3.3.2 Crescimento de Regiões Modificado $\quad 80$

3.4 Método de Canny-Watersheds 84

3.4.1 Segmentação $\quad 85$

3.4.2 Pós-processamento $\quad 86$

$\begin{array}{ll}3.5 \text { Classificação } & 90\end{array}$

4 Resultados e Discussão $\quad 92$

4.1 Otimização do ângulo de polarização linear $\quad 92$

4.1.1 Análise dos Cristais 93

4.2 Comparação do CRM na segmentação de imagens CPOL com LPOL 96

4.2.1 Análise dos Cristais $\quad 98$

4.2.2 Análise das classes $\quad 99$ 
4.3 Comparação do método CRM com CW 102

$\begin{array}{lll}\text { 4.3.1 Análise dos Cristais } & 104\end{array}$

$\begin{array}{ll}\text { 4.3.2 Análise das classes } & 104\end{array}$

4.4 Classificação 108

4.4.1 Classificação método CRM das imagens CPOL 108

4.4.2 Classificação método CRM das imagens LPOL 111

5 Conclusão e Trabalhos Futuros $\quad 115$

A Artigo da Tese Publicado em Periódico 129 


\section{Lista de figuras}

2.1 Evolução da Produção Mundial de Aço Bruto no período 1950 2010.[23]

2.2 Hematita: (a) e (b) Imagens de fragmentos do mineral; (c) Estrutura trigonal-hexagonal visualizando os planos; (d) Modelo de bolas e varetas da cela unitária.[4, 31]

2.3 Variação de brilho entre dois cristais adjacentes: (a) Imagem em campo claro; (b) Imagem com polarização de um mesmo campo.[67] 27

2.4 Luz natural com os diferentes comprimentos de onda correspondentes a cada cor. Neste caso só foi representada a intensidade do campo elétrico para facilitar a visualização.[69]

2.5 Propagação de uma onda plana linearmente polarizada. Neste caso só foi representada a intensidade do campo elétrico para facilitar a visualização.[70]

2.6 Propagação de uma onda com polarização circular. Neste caso só foi representada a intensidade do campo elétrico para facilitar a visualização.[71]

2.7 Variação de brilho entre cristais adjacentes: (a) Imagem em campo claro; (b) Imagem com polarização linear; (c) Imagem com polarização circular, de um mesmo campo.

2.8 Sequência padrão de PADI por etapas.[52]

2.9 Possíveis transformações em registro de imagens.[52]

2.10 Possíveis pontos de controle: (a) Imagem de referência e (b) Imagem a ser registrada; (c) Imagem registrada.

2.11 Função de transformação para o realce do brilho nas bordas dos objetos.[90]

2.12 Delineação: (a) Imagem original, seu histograma e uma visão ampliada da região demarcada em vermelho; (b) Imagem delineada, seu histograma e a ampliação da mesma região demarcada em vermelho.[76]

2.13 Exemplo de um filtro mediana de tamanho $3 \times 3$.

2.14 Uso do filtro mediana: (a) Imagem esquemática com ruído sal-epimenta; (b) Imagem esquemática com ruído reduzido pelo filtro mediana de tamanho $3 \times 3$.[80]

2.15 Exemplo de limiarização bimodal: (a) Imagem original; (b) Imagem binária; (c) Tom de corte no histograma.

2.16 Exemplo de limiarização pentamodal: (a) Imagem original em 256 tons de cinza; (b) Imagem quinária com as fases diferençadas com cores; (c) Tons de corte no histograma.[76]

2.17 Exemplo de detecção de bordas pelo método de Canny: (a) Imagem original; (b) Imagem binária com as bordas dos cristais de hematita. 45

2.18 Exemplo de crescimento de regiões com $t=3$ : (a) Imagem em tons de cinza; (b) Duas regiões $\left(R_{1}\right.$ e $\left.R_{2}\right)$ separadas com $n=2$ sementes (em vermelho); (c) Três regiões $\left(R_{1}, R_{2}\right.$ e $\left.R_{3}\right)$ separadas com $n=3$ sementes (em vermelho). 
2.19 Operações lógicas pixel a pixel.

2.20 Operações lógicas básicas (NOT, OR e AND). 49

2.21 Operação lógica XOR. $\quad 50$

2.22 Erosão e Dilatação. $\quad 52$

2.23 Abertura e Fechamento. 53

2.24 Exemplo esquemático do uso de MDE: (a) Imagem binária; (b) Imagem MDE; (c) Erosão de passo 2.

2.25 Método dos divisores de água: (a) Imagem binária inicial; (b) Imagem MDE; (c) Imagem sementes; (d) Imagem binária com os objetos separados.

2.26 Separação de objetos numa imagem binária dependendo do tipo de conectividade entre pixels: (a) Imagem binária inicial; (b) Objetos separados com conectividade 4; (c) Objetos não separados com conectividade 8.[117]

2.27 Cálculo do perímetro de um objeto através dos passos horizontais, verticais e diagonais.

2.28 Comparação de fatores adimensionais de forma.[116]

2.29 Tipos de sistemas de classificação utilizando os parâmetros característicos FFC e RA: (a) Classificação supervisionada; (b) Classificação não-supervisionada.

3.1 Três imagens de um mesmo campo: (a) Imagem SP; (b) Imagem LPOL com polarizador girado $+10^{\circ}$; (c) Imagem LPOL com polarizador girado $-10^{\circ}$ e; (d) Imagem CPOL.

3.2 Influência do ângulo de polarização no contraste dos cristais.[65] 74

3.3 Fluxograma do pré-processamento.

3.4 Correção do gradiente de iluminação.

3.5 Registro de duas imagens de um mesmo campo: (a)-(b) Imagens LPOL e CPOL sem registar; (c)-(d) Imagens LPOL e CPOL registradas e; (e)-(f) Imagens $L P O L$ e CPOL registradas e com a borda preta eliminada.

3.6 Correção da subexposição nas imagens CPOL e LPOL: (a) Imagem CPOL com subexposição; (b) Imagem LPOL com subexposição; (c) Imagem CPOL com correção da subexposição e; (d) Imagem LPOL com correção da subexposição.

3.7 Problemas gerados na imagem CPOL ao corrigir o gradiente de iluminação com uma imagem de fundo inadequada: (a) Canto inferior esquerdo da imagem CPOL ampliado e (b) Canto superior direito da imagem CPOL ampliado.

3.8 Fluxograma da segmentação pelo método CRM.

3.9 Segmentação da hematita: (a) Imagem SP; (b) Imagem binária da hematita segmentada; (c) Imagem CPOL com hematita e restos de fases espúrias e; (d) Imagem CPOL com hematita isolada.

3.10 Segmentação grosseira dos cristais de hematita: (a) Imagem CPOL normal; (b) Imagem CPOL com ruído gaussiano; (c) Imagem resultante do Canny sobre a imagem normal; (d) Imagem resultante do Canny sobre a imagem com ruído; (e) Cristais de hematita segmentados através do Canny e; Cristais de hematita grosseiramente segmentados através do Canny. 
3.11 Segmentação final dos cristais de hematita: (a) Imagem binária resultante do divisor de águas; (b) Imagem binária com as sementes; (c) Cristais de hematita finalmente segmentados e; (d) Imagem colorida para ajudar na visualização dos cristais de hematita.

3.12 Fluxograma da segmentação pelo método CW.

3.13 Canny da imagem CPOL: (a) Canal vermelho da imagem CPOL; (b) Canal verde da imagem CPOL; (c) Canal azul da imagem CPOL; (d) Canny da imagem do canal vermelho; (e) Canny da imagem do canal verde; (f) Canny da imagem do canal azul e; (g) Soma dos três Canny.

3.14 Fluxograma do pós-processamento do método CW.

3.15 Sequência de pós-processamento para eliminar objetos espúrios na imagem segmentada.

3.16 Método do divisor de águas usado para corrigir bordas incompletas do Canny.

4.1 Possível causa da discrepância do feret mínimo no campo 4: (a) Imagem LPOL com arranhão; (b) Imagem automaticamente segmentada mantendo o arranhão e; (c) Imagem manualmente segmentada com arranhão eliminado.[65]

4.2 Distribuições do feret mínimo das segmentações manual e automática para os campos 1 e 4.[65]

4.3 Comparação CPOL - LPOL: (a) Imagem CPOL original; (b) Imagem LPOL original; (c) Imagem CPOL segmentada; (d) Imagem LPOL segmentada; (e) Imagem CLUT dos cristais CPOL e; ( $f$ ) Imagem CLUT dos cristais LPOL.

4.4 Histogramas referente ao feret mínimo dos cristais segmentados pelo método CRM nas classes de hematita para CPOL e LPOL.

4.5 Histogramas referente ao feret máximo dos cristais segmentados pelo método CRM nas classes de hematita para CPOL e LPOL.

4.6 Comparação dos cristais obtidos por CW com os obtidos por CRM:

(a) Imagem CPOL original; (b) Cristais segmentados pelo CRM; (c) Cristais segmentados pelo CW; (d) Cristais segmentados pelo CRM com CLUT e; (e) Cristais segmentados pelo CW com CLUT. 102

4.7 Histogramas referente ao feret mínimo dos cristais segmentados pelos métodos CRM e CW nas classes de hematita para CPOL.

4.8 Histogramas referente ao feret máximo dos cristais segmentados pelos métodos CRM e CW nas classes de hematita para CPOL. 


\section{Lista de tabelas}

2.1 Reservas mundiais de minério de ferro no ano $2011\left(10^{6} \mathrm{t}\right) \cdot[18] \quad 19$

2.2 Reservas brasileiras de minério de ferro Medidas e Indicadas (em toneladas).[20]

2.3 Produção Mineral (em milhões de tons/ano).[19]

2.4 Quadro ilustrativo com as principais morfologias de cristais de óxidos/hidróxidos de ferro.[29]

2.5 Orientações estruturais entre os óxidos de ferro.[4]

4.1 Otimização do ângulo de polarização.[65]

4.2 Comparação entre os resultados da segmentação automática e manual dos cristais de hematita.[65]

4.3 Comparação entre os resultados das medidas dos cristais segmentados pelo método CRM nas imagens CPOL e LPOL.

4.4 Resultado das medidas dos cristais segmentados pelo método CRM nas classes de hematita para CPOL e LPOL.

4.5 Comparação entre os resultados das medidas dos cristais segmentados pelos métodos CRM e CW nas imagens CPOL.

4.6 Resultado das medidas dos cristais segmentados pelos métodos CRM e CW nas classes de hematita para CPOL.

4.7 Classificação das classes nas, imagens CPOL segmentadas pelo método CRM, usando todos os atributos.

4.8 Classificação das classes, nas imagens CPOL segmentadas pelo método CRM, usando PCA de 5 componentes.

4.9 Classificação das classes, nas imagens CPOL segmentadas pelo método CRM, usando FDA de 2 componentes.

4.10 Matriz de confusão da autovalidação do classificador quadrático.

4.11 Classificação das classes nas, imagens LPOL segmentadas pelo método CRM, usando todos os atributos.

4.12 Classificação das classes, nas imagens LPOL segmentadas pelo método CRM, usando PCA de 5 componentes.

4.13 Classificação das classes, nas imagens LPOL segmentadas pelo método CRM, usando FDA de 2 componentes.

4.14 Matriz de confusão da autovalidação do classificador quadrático. 


\title{
Lista de Abreviaturas
}

\author{
ADI - Análise Digital de Imagens \\ BIF - Banded Iron Formation \\ CCD - Charge-Coupled Device
}

CETEM - Centro de Tecnologia Mineral

CPOL - Polarização Circular

CRM - Crescimento de Regiões Modificado

CW - Canny-Watersheds

DEMa - Departamento de Engenharia de Materiais

FDA - Análise Discriminante de Fisher

GPU - Graphics Processing Unit

KLT - Transformada de Karhunen-Loève

LDA - Análise Discriminante Linear

LMD - Laboratório de MD da PUC-Rio

LPOL - Polarização Linear

CLUT - Colour Look-Up table (Tabela de visualização)

MCTI - Ministério da Ciência, Tecnologia e Inovação

MD - Microscopia Digital

MDE - Mapa de Distâncias Euclidianas

MDS - Escalonamento Multidimensional

MLR - Microscópio de Luz Refletida

PADI - Processamento e Análise Digital de Imagens

PCA - Análise de Componentes Principais

PDI - Processamento Digital de Imagens

RGB - Sistema de cores Red, Green, Blue

SCT - Setor de Caracterização Tecnológica

SIFT - Scale Invariant Feature Transform

SP - Sem Polarização

TA - Taxa de Acerto

UNCTAD - Conferência das Nações Unidas para o Comércio e o Desenvolvimento 